\title{
Dynamical Electro-Optical Characterization of Policryps Gratings
}

\author{
G. AbBate*, A. Marino and F. Vita \\ INFM UdR of Naples and DSF Università di Napoli Federico II, Naples, Italy
}

\begin{abstract}
We have studied the dynamical response at 633 and $1550 \mathrm{~nm}$ of a new polymer-liquid crystal composite, realized in a form of a thick grating. This composite is known as Policryps, an acronym that stands for "Polymer Liquid Crystals Polymer Slides", because it shows a sequence of polymeric sheets alternated by layers containing only liquid crystal. Policryps gratings can be electrically switched between diffracting and non-diffracting states with a good diffraction efficiency in the visible region. After optimization of their performances, including response time, in the near infrared region, they can be conveniently proposed as a key component in photonic devices for telecom applications. Our results show very interesting response times ranging from 1 to $3 \mathrm{~ms}$ (switching on) and from 0.1 to $0.4 \mathrm{~ms}$ (switching off). Furthermore, switching ON times appear to be ruled by two different physical mechanisms with characteristic times differing by one order of magnitude. A deeper insight of these mechanisms might lead to response times, all in the submillisecond range.
\end{abstract}

PACS numbers: $42.70 . \mathrm{Df}, 42.79 . \mathrm{Kr}$

\section{Introduction}

A switchable diffraction grating is a key component for the realization of several devices used in the chain of optical communication networks, such as switches, add-and-drop systems, beam deflectors, routers [1]. For instance, one of the easiest way to realize an optical multiplexing/demultiplexing device is to integrate one or several switchable gratings in a guiding system such as a fiber or a waveguide. A number of different technologies have been proposed for realizing such a component, including, quite recently, a technology based on liquid crystals (LC) materials [2].

* corresponding author; e-mail: giancarlo.abbate@na.infn.it 
Recently an innovative process has been proposed for the realization of diffraction gratings with polymer-LC composites [3, 4]. The resulting material has been named by the authors Policryps, that is "POlymer LIquid CRYstals Polymer Slides". It is composed by a sequence of homogeneously aligned LC layers separated by isotropic polymer walls having approximately the same thickness of the LC ones.

Due to their morphology, being a stack of uniform and transparent layers, Policryps samples are inherently transparent. Just few electro-optical studies of this material have been until now performed [3-5]. However, this first characterization gives a clear indication that very high diffraction efficiency (DE) can be reached while the driving voltage can be kept at reasonable values: in a few words, Policryps seems to be a very attractive material and it is well worth continuing the research studies on this composite in order to fully characterize it and eventually to get feedback for its optimization at telecom wavelengths.

The aim of the present work is to give a dynamical characterization of some Policryps samples both in the visible range, at $\lambda_{\text {red }}=633 \mathrm{~nm}$, and in the near infrared (NIR), at $\lambda_{\mathrm{NIR}}=1550 \mathrm{~nm}$, that is the C-band of the 3rd window of optical communications.

\section{Policryps operating model}

The samples we have studied have been kindly provided by the group that has invented Policryps at the University of Calabria, in the frame of a running Italian National Research Project [6]. We got two different samples labeled as HCB-25 and HCB-31. They are made by a composition of Norland Optical Adhesive NOA-65, diluted in a $30 \%$ concentration of $5 \mathrm{CB}$ nematic LC, commercially available by Merck (Germany): the grating length was a few millimeters and the pitch $\Lambda$ was $1.34 \mu \mathrm{m}$ for both samples, while the grating depth $d$ was $7.8 \mu \mathrm{m}$ and $8.8 \mu \mathrm{m}$, respectively. The whole production process of Policryps is presently patent
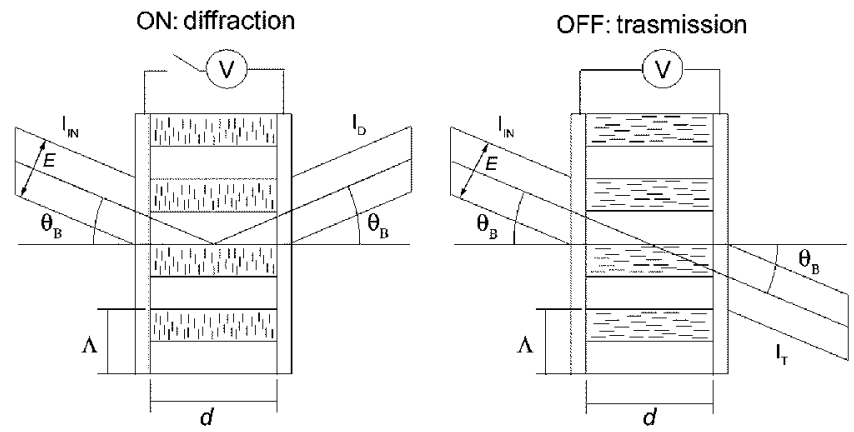

Fig. 1. Policryps operating model. 
pending. Policryps samples were sandwiched between glass plates and sealed. The glass plates were ITO coated for applying an electric field to the cell and no further alignment treatment was made. The studied geometry is sketched in Fig. 1: Policryps shows itself as a thick grating, thus exhibiting diffraction according to the Bragg law [7].

By applying a proper electric field, we can switch the grating among the ON and OFF states. Without any applied voltage, a linearly polarized incident light beam will see the phase grating formed by the two materials with different refractive index and it will be diffracted. Applying an external voltage, the molecular director in the LC slides can be reoriented changing the refractive index seen by the impinging light. Eventually, we can reach the condition $n_{\mathrm{LC}}=n_{\text {Polymer }}$; if and when this condition is met, the beam is no more diffracted (Fig. 1) ${ }^{\dagger}$.

Policryps grating behavior is strictly polarization dependent: it is possible to observe a diffracted beam only for a $p$-polarized wave. The reason should be found in the molecular orientation inside the LC layers, schematically represented in Fig. 1. In the absence of applied electric field, we can reasonably assume [5] that the LC molecular director is oriented parallel to the glass plates and perpendicular to the polymeric sheets. When the field is applied the LC molecular director is realigned parallel to the electric field, owing to the positive dielectric anisotropy of the nematic 5CB. A nematic LC can be optically viewed as a uniaxial crystal with the optic axis direction coinciding with the molecular director. Thus, a $p$-polarized light beam impinging at normal incidence on the sample will experience the extra-ordinary value of the LC refractive index, $n_{e}$, without applied field, and the ordinary value, $n_{\mathrm{o}}$, with applied field. Now, let us recall that at $\lambda_{\text {red }}$ and $25^{\circ} \mathrm{C}$ the polymer NOA-65 refractive index is $n_{\mathrm{p}}=1.56$ and the values for $5 \mathrm{CB}$ indices are $n_{\circ}=1.53$ and $n_{\mathrm{e}}=1.71$, respectively. As previously said, Policryps samples in our experimental geometry (see Fig. 1) work in the Bragg regime. Accordingly, the maximum $\mathrm{DE}$ condition is reached when the impinging beam is at the Bragg angle and not at normal incidence. Thus, the actual LC refractive index seen by a $p$-wave propagating in it is dependent on the angle $\theta$ between the light polarization direction and the optic axis, namely $\bar{n}(\theta)=n_{\mathrm{o}} n_{\mathrm{e}} /\left[n_{0}^{2} \cos ^{2}(\theta)+n_{\mathrm{e}}^{2} \sin ^{2}(\theta)\right]^{1 / 2}$, and is always smaller than $n_{\mathrm{e}}$ without field and greater than $n_{\mathrm{O}}$ with applied field. However, at $\lambda_{\text {red }}$ our Bragg angle was around $13^{\circ}$ and the corresponding $\bar{n}(\theta)$ values are quite close to $n_{\mathrm{e}}$ and $n_{\mathrm{o}}$, respectively. In conclusion, using the materials' optical parameters at $\lambda_{\text {red }}$, a high index contrast is exhibited by the grating layers to the propagating light when no field is applied and a much smaller one when the electric field is on. As a consequence, a high DE is expected without field and almost no DE with electric field.

†Of course, in a real system this condition can be fulfilled more or less accurately depending on the choice of material parameters, temperature range, and working wavelength: this is clearly demonstrated in Sec. 3. 


\section{Experimental}

At $\lambda_{\text {red }}$, as expected by the discussion in the previous section, we actually observed over $90 \% \mathrm{DE}$ and switching off, almost at $0 \%$ diffraction, with an applied voltage of $55 \mathrm{~V}$. On the contrary, using NIR light, a much lower DE less than $20 \%$ was observed; moreover, at $\lambda_{\text {NIR }}$ a complete switching off is reached for an applied voltage of about $45 \mathrm{~V}$ and increasing it further leads to DE increasing again to a few percent. This result is not unexpected because our samples were produced for having optimized performances with red light: we have reported and commented it extensively in a previous paper [5], while in the present one we focus our discussion on the dynamical response of Policryps grating to a variable electric field. We performed all our measurements on both samples. The obtained results are quite similar, thus we report here only those for HCB-25. In all the following, the impinging light is always $p$-polarized. Policryps response times were measured by recording the diffracted beam signal detected by a photodiode. A driving voltage, enough to completely switch off the diffractive device, was applied to the ITO electrodes, using different frequencies in the range of 1-100 Hz. In order to avoid static electric biasing of the sample, we always took care of applying a zero-average waveform. Figure 2 shows the oscilloscope image recorded with $\lambda_{\text {red }}$ light at voltage frequencies of $100 \mathrm{~Hz}$ (a) and $1 \mathrm{~Hz}$ (b), respectively. The applied waveform is shown in the lower part and the optical response in the upper part.

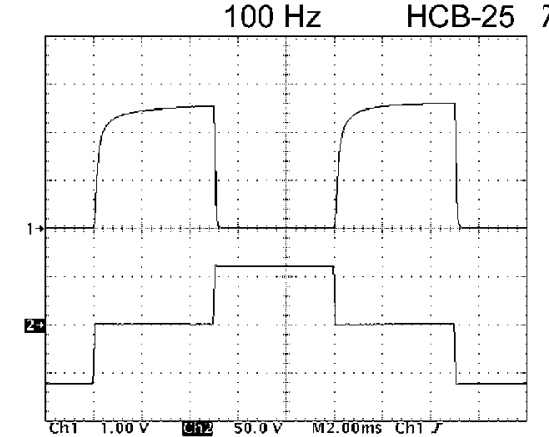

a)

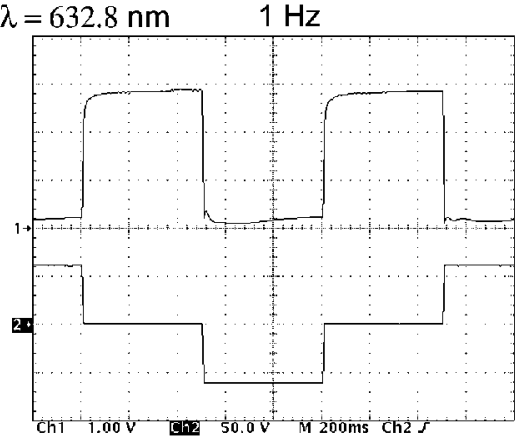

b)

Fig. 2. Optical response, upper curves, and driving waveform, lower curves, at $633 \mathrm{~nm}$; (a) voltage frequency $100 \mathrm{~Hz}$, (b) $1 \mathrm{~Hz}$.

Figure 3 shows the analogous images recorded with $\lambda_{\text {NIR }}$ light. In Figs. $2 \mathrm{~b}$ and $3 \mathrm{~b}$, the optical response at $1 \mathrm{~Hz}$ exhibits an irregular behavior and even a slight increase with time in the detected signal during the half period corresponding to the OFF state, that is when the applied voltage is different from zero. This was unexpected and probably due to a molecular flow and/or turbulence induced by free charge (ions) motion under the effect of a constant voltage for a quite long 


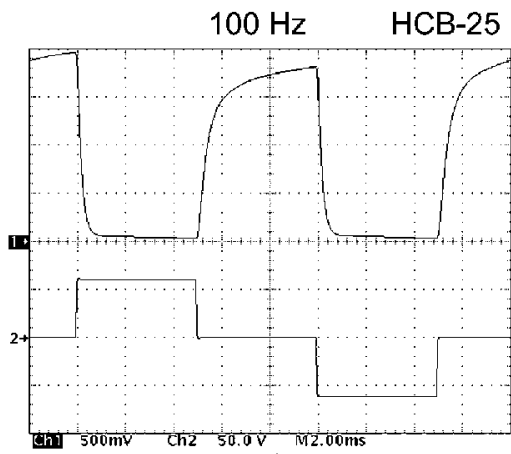

a)

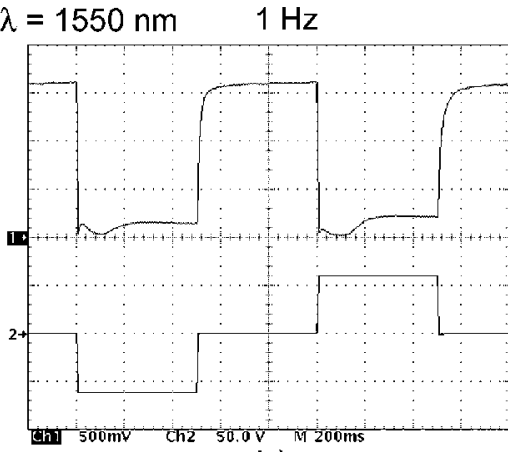

b)

Fig. 3. Optical response, upper curves, and driving waveform, lower curves, at $1550 \mathrm{~nm}$; (a) voltage frequency $100 \mathrm{~Hz}$; (b) $1 \mathrm{~Hz}$.

time, say $500 \mathrm{~ms}$. To overcome this problem, we used a different waveform, in which the previous square voltage at a slow frequency $f_{1}$ is further modulated at a faster frequency $f_{2}$, in the range of $1-10 \mathrm{kHz}$, the voltage amplitude being fixed at $60 \mathrm{~V}$. Generally, liquid crystals have response times too slow to follow such fast driving signal as the ones at frequency $f_{2}$, so that the reorientation torque is almost equivalent to that imposed by a constant voltage of the same rms value. However, in this way we can get rid of any effect caused by collective ion motion inside the sample.

In Figs. 4 and 5, we report the same pictures captured on the oscilloscope display as in Figs. 2 and 3 , but with the double frequency modulation of the waveform ${ }^{\ddagger}$.

A summary of the most significant results is presented in the Table. Differences among the achievements at $\lambda_{\text {red }}$ and $\lambda_{\text {NIR }}$ are again evident, despite the fact that molecular reorientation in LC is obviously only driven by electric and elastic forces and must not depend on the light traveling in the sample. However, the response of the diffraction grating is not immediately and only related to the LC molecular director reorientation. The diffraction efficiency of a Bragg grating is ruled by the so-called Kogelnik formula ${ }^{\hat{s}}[7]$ :

$$
D E=\sin ^{2} \frac{2 \pi d \Delta n}{\Lambda \cos \vartheta_{\mathrm{B}}},
$$

where $\vartheta_{\mathrm{B}}$ is the Bragg angle, $d$ is the grating depth, $A$ - the grating pitch and $\Delta n$ - the difference between the polymer and the LC refractive index. The most important physical parameter affecting DE value in this formula is the refractive

\footnotetext{
$\ddagger$ Notice that in Figs. $4 \mathrm{~b}$ and $5 \mathrm{~b}$, recorded with $f_{1}=1 \mathrm{~Hz}$, the modulation at $f_{2}$ is not viewable in the waveform, because the ratio $f_{2} / f_{1}$ is too high to let both frequencies be displayed simultaneously; for the same reason the shown amplitude scale (channel 2) is meaningless.

$\S$ For anisotropic materials Kogelnik formula has been extended by the theory of Montemezzani and coworkers [8].
} 


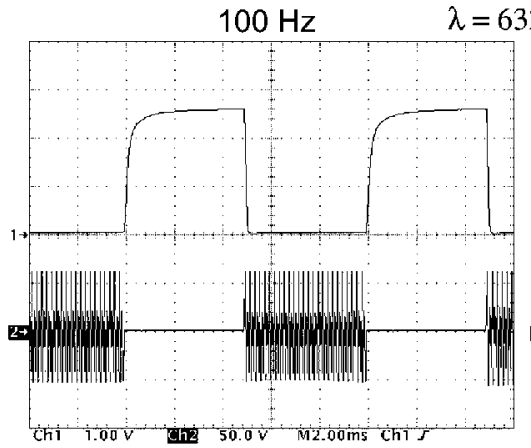

a)

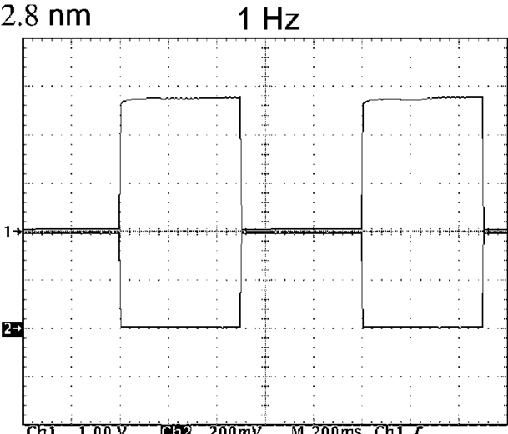

b)

Fig. 4. Optical response, upper curves, and new driving waveform, lower curves, at $633 \mathrm{~nm}$; (a) voltage frequency $100 \mathrm{~Hz}$, (b) $1 \mathrm{~Hz}$.

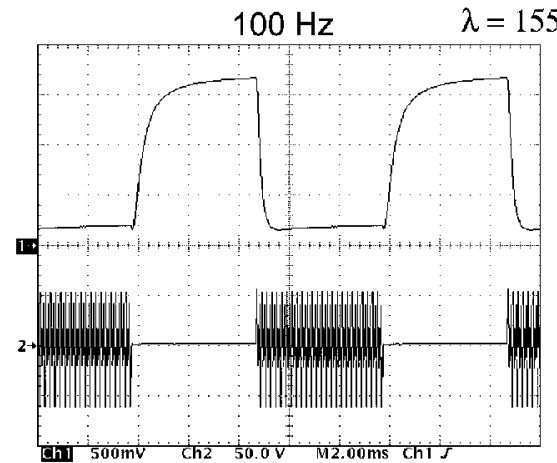

a)

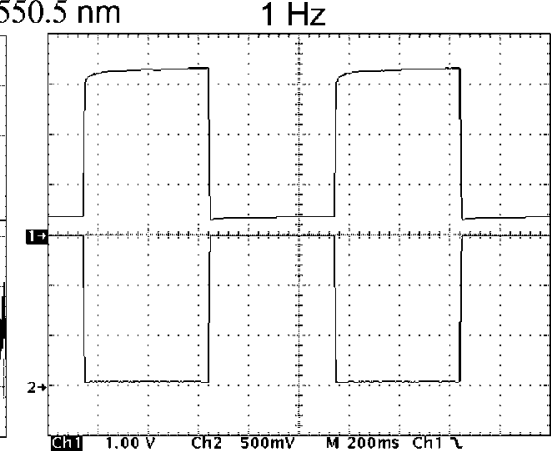

b)

Fig. 5. Optical response, upper curves, and new driving waveform, lower curves, at $1550 \mathrm{~nm}$; (a) voltage frequency $100 \mathrm{~Hz}$, (b) $1 \mathrm{~Hz}$.

index contrast of the grating. Unfortunately, not many data about refractive index values at $\lambda_{\mathrm{NIR}}$ are presently available for LC materials, so that a comprehensive comparison of theoretical predictions and experimental findings has not been yet performed. However, this study is now in progress, including measurements of refractive index values at $\lambda_{\text {NIR }}$ [9], because, in our opinion, it is crucial for a correct design of Policryps gratings (and in general of LC-based devices) optimized for working at NIR wavelengths.

From the last two columns of the Table, we notice also that $t_{\mathrm{OFF}}$ times are one order of magnitude smaller than $t_{\mathrm{ON}}$ times, the former being in the hundreds of $\mu$ s range, and the latter in the ms one. Let us recall that the grating is switched OFF while the electric field is applied and switched ON when it is removed. For that, the torque responsible for molecular reorientation in the LC layers is ruled during switching OFF by the balance between elastic forces and electric ones. In 
TABLE

Response times of Policryps samples.

\begin{tabular}{c|c|c|c|c}
\hline \hline $\begin{array}{c}\text { Light wa velength } \\
\lambda[\mathrm{nm}]\end{array}$ & $\begin{array}{c}\text { Voltage frequency } \\
f_{1}[\mathrm{HZ}]\end{array}$ & $\begin{array}{c}\text { Modulation frequency } \\
f_{2}[\mathrm{~Hz}]\end{array}$ & $t_{\text {ON }}[\mu \mathrm{s}]$ & $t_{\text {OFF }}[\mu \mathrm{s}]$ \\
\hline 633 & 1 & 1000 & 1300 & 160 \\
633 & 10 & 1000 & 1000 & 130 \\
633 & 100 & 10000 & 900 & 70 \\
1550 & 1 & 1000 & 2800 & 440 \\
1550 & 10 & 10000 & 3200 & 320 \\
1550 & 50 & 5000 & 1500 & 370 \\
1550 & 100 & 10000 & 1300 & 320
\end{tabular}

our case, the electric field is quite intense, that is about $4.5 \mathrm{~V} / \mu \mathrm{m}$, yielding a fast reorientation. On the other hand, molecular reorientation during switching $\mathrm{ON}$ is only due to restoring elastic forces, that is mainly to surface interactions with the polymer layers and the glass substrates. Thus, the observed difference in response times is in agreement at least qualitatively with the physical picture underlying it. However, we observed large fluctuations in the measured $t_{\mathrm{ON}}$ times, not comparable with those observed in $t_{\mathrm{OFF}}$ ones. For instance, standard errors in $t_{\mathrm{OFF}}$ have been estimated to be about $5 \%$, while standard errors in $t_{\mathrm{ON}}$ are even greater than $25 \%$, putting some doubts on the way we have measured them, as the time interval between $10 \%$ and $90 \%$ values of the maximum light signal detected by the photodiode. Looking more carefully at the switching ON process, it appears that it is not ruled by a single time constant. Figures 6 and 7 show digitized data of switching ON oscilloscope tracks, open circles, together with a single exponential

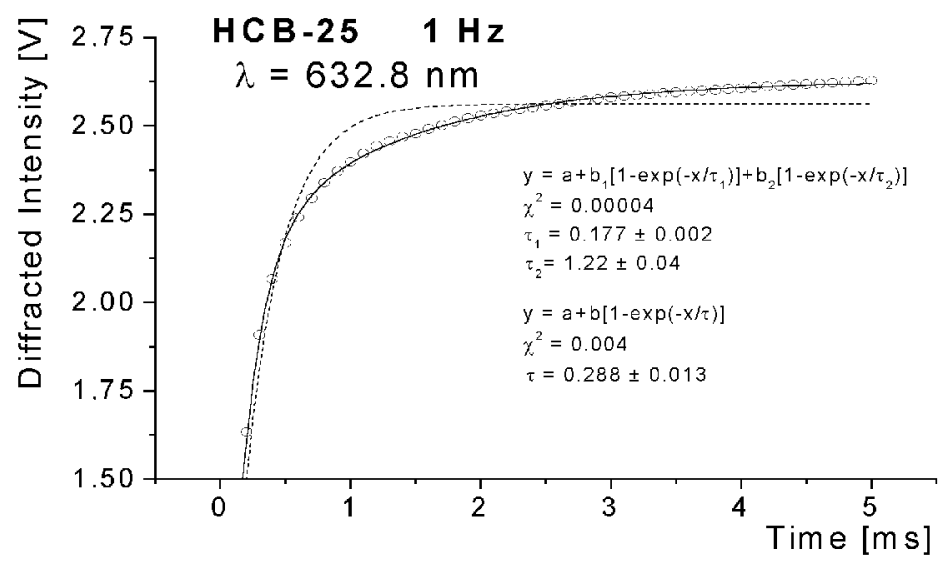

Fig. 6. Analysis of optical response in the $\mathrm{OFF}-\mathrm{ON}$ transition at $633 \mathrm{~nm}$ : digitized data (open circles); single exponential fit (dotted line); two-exponential fit (solid line). 


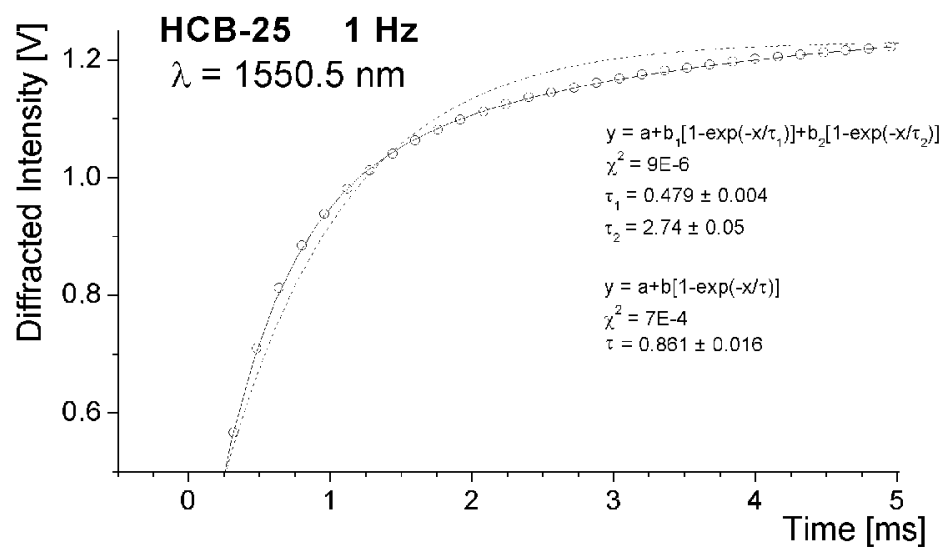

Fig. 7. Analysis of optical response in the $\mathrm{OFF}-\mathrm{ON}$ transition at $1550 \mathrm{~nm}$ : digitized data (open circles); single exponential fit (dotted line); two-exponential fit (solid line).

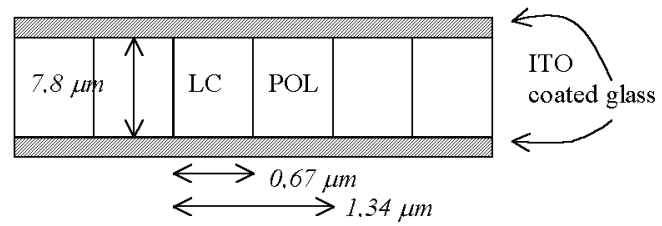

Fig. 8. Geometric dimensions of the grating (not in scale).

fit, dotted lines, and a two-exponential fit, solid lines. The figures correspond to two properly chosen experimental recordings, but all the others gave completely similar results. It is fairly evident that the single exponential curve cannot fit reasonably the data points, while a very good fit is obtained by the two-exponential curve. In fact, a $\chi^{2}$ value 100 times lower is obtained in the latter case, see the inserts in Figs. 6 and 7 . This result gives us an indication that two competing physical mechanisms are intervening in restoring the orientational order in the LC layers: are both of them of elastic nature? An answer to this question and a correct individuation of which mechanisms are involved in this process needs further investigations, especially systematic experimental measurements, but also a proper theoretical model for it. We should limit ourselves at this stage to point out that the geometry of the LC regions in Policryps is very different from the geometry in a common LC cell, where only a 1-dimensional confinement is performed. Figure 8 shows clearly how LC layers in Policryps are confined in 2-D: in one dimension between the glass substrates, and in the other between the polymer sheets. However, the length scale is not the same in both dimensions, being about $8 \mu \mathrm{m}$ in one and about $0.7 \mu \mathrm{m}$ in the other. Thus, the ratio between characteristic 
lengths in Policryps is of the same order as the ratio between the two measured time constants (shown in the inserts of Figs. 6 and 7). If further studies will prove that these ratios are correlated, and when we shall have a more precise idea of the physical mechanisms involved, then we could properly act on the anchoring energy of different surfaces, for instance applying proper surfactants, in order to let the smaller time constant be the only one relevant in the switching ON process. Thus, our goal is getting a sub-millisecond grating switch, in particular at $\lambda_{\mathrm{NIR}}$, which will render Policryps a very interesting component in the fabrication of photonic devices.

\section{Conclusions}

We performed a dynamical characterization of the first produced Policryps gratings, undergoing an electric field variable in time that permitted switching ON and OFF of the diffracted beam. In particular, we pointed out differences between experimental findings using red light and NIR light in the telecom C-band. Our present aim is combining these results with theoretical models for anisotropic diffraction gratings and with refractive index measurements at $\lambda_{\mathrm{NIR}}$ in order to design and realize Policryps optimized for telecom applications.

The observed response times, ranging from 1 to $3 \mathrm{~ms}$ for switching $\mathrm{ON}$ and from 0.1 to $0.4 \mathrm{~ms}$ for switching $\mathrm{OFF}$, are already very attractive for practical applications. Moreover, Policryps gratings, exhibiting a more complex confining of LC material than conventional LC cells, are interesting systems also for fundamental studies on elastic bulk and surface interactions in liquid crystals. Our results on the double time constant behavior of the switching ON process can be a starting point for further investigations in that direction.

\section{Acknowledgments}

This work has been performed under the Italian Ministry of Education and Research (MIUR) project PRIN2000 [7]. We gratefully acknowledge useful discussions with our partners of Rome and Cosenza groups in that project. In particular, Prof. C. Umeton group in Cosenza has provided all the samples investigated here. Moreover, Prof. Umeton and Dr. Roberto Caputo gave us a number of very important hints during many discussions.

\section{References}

[1] L. Eldada, Opt. Eng. 40, 1165 (2001) and references therein.

[2] Liquid Crystals V, Ed. I.C. Khoo, Proc. SPIE 4463 (2001); the whole volume is dedicated to the subject.

[3] R. Caputo, A.V. Sukhov, C. Umeton, R.F. Ushakov, J. Exp. Theor. Phys. 91, 1190 (2000). 
[4] R. Caputo, A.V. Sukhov, N.V. Tabirian, C. Umeton, R.F. Ushakov, Chem. Phys. 271, 323 (2001).

[5] G. Abbate, A. Marino, F. Vita, to be published in Mol. Cryst, Liq. Cryst.

[6] Devices for Routing in Optical Networks Using New Liquid Crystals Materials and Composites; Research Project of National Interest (PRIN 2000) by Italian Ministry of Education and Research.

[7] T.K. Gaylord, M.G. Moharam, Proc. IEEE 73, 894 (1985).

[8] G. Montemezzani, M. Zgonik, Phys. Rev. E 55, 1035 (1997).

[9] G. Abbate, G. Del Gais, L. De Stefano, A. Marino, T. Wagner, to be published in Mol. Cryst. Liq. Cryst. 